\title{
Performance Analysis of IEEE 802.15.4 Compliant Wireless Devices for Heterogeneous Indoor Home Automation Environments
}

\author{
Juan Antonio Nazabal, Peio López Iturri, Leire Azpilicueta, \\ Francisco Falcone, and Carlos Fernández-Valdivielso \\ Electrical and Electronic Engineering Department, Public University of Navarre, Campus de Arrosadia, 31006 Pamplona, Spain
}

Correspondence should be addressed to Francisco Falcone, francisco.falcone@unavarra.es

Received 27 June 2012; Accepted 18 September 2012

Academic Editor: Tat Yeo

Copyright (C) 2012 Juan Antonio Nazabal et al. This is an open access article distributed under the Creative Commons Attribution License, which permits unrestricted use, distribution, and reproduction in any medium, provided the original work is properly cited.

\begin{abstract}
The influence of topology as well as morphology of complex indoor scenarios in the deployment of wireless sensor networks and wireless systems applied to home and building automation systems is analyzed. The existence of loss mechanisms such as material absorption (walls, furniture, etc.) and strong multipath components as well as the increase in the number of wireless sensors within indoor scenarios increases the relevance in the configuration of the heterogeneous wireless systems. Simulation results by means of empirical-based models are compared with an in-house 3D ray launching code as well as measurement results from wireless sensor networks illustrate the strong influence of the indoor scenario in the overall performance. The use of adequate radioplanning strategies lead to optimal wireless network deployments in terms of capacity, quality of service, and reduced power consumption.
\end{abstract}

\section{Introduction}

The use of wireless sensor networks (WSNs) is growing rapidly into a large number of fields of application [1$3]$, such as building automation systems (BAS), industrial monitoring $[4,5]$, farming and agriculture [6-9], structural monitoring [10], civil infrastructure state monitoring [11$13]$, healthcare monitoring $[14,15]$, location and guiding [16], or security and defense, among others. The future trend is to increase the number of nodes, in order to increase the interaction with users as well as capturing more information from the surrounding environment. On the other hand, the use of building automation systems, especially oriented to home automation, is growing all over the world [17-22]. As time goes by more homes integrate an automation system for intelligently controlling elements such light, heating, and energy management. The connection between the components of these systems has been made typically via electric conducting cables but nowadays the use of wireless technologies for communication with each other is taking a predominant role.

In the case of home and building automation systems, the foreseen scenario is indoor, with high complexity related to the topology as well as the morphology. This leads to increased losses, in terms of material absorption as well as to strong multipath propagation. Future systems will tend to use a large number of sensors, in which energy consumption as well as capacity are key issues to be considered.

The application of Building Automation systems to residential environments is also called Smart Home systems. In this way, a Smart Home can be defined as the application of automation and integration systems of housing facilities to improve the daily life of final users, which are usually domestic. This technology has been under development during many years with different standards and technologies such as KNX, BACnet, or LON allowing the development of different applications and systems in the last few years [2326]. All Smart home networks are based on communication 


\begin{tabular}{|ccccccc} 
Frequency & Band & Coverage & Data rate & $\begin{array}{c}\text { Number of } \\
\text { channels }\end{array}$ & $\begin{array}{c}\text { Rx } \\
\text { sensitivity }\end{array}$ & Modulation \\
\hline $2.4 \mathrm{GHz}$ & IMS & Worldwide & $250 \mathrm{Kbps}$ & 16 & $-85 \mathrm{dBm}$ & O-QPSK \\
\hline $868 \mathrm{MHz}$ & IMS & Europe & $20 \mathrm{Kbps}$ & 1 & $-92 \mathrm{dBm}$ & BPSK \\
\hline $915 \mathrm{MHz}$ & IMS & America & $40 \mathrm{Kbps}$ & 10 & $-92 \mathrm{dBm}$ & BPSK \\
\hline
\end{tabular}

FIGURE 1: IEEE 802.15.4 radio characteristics.

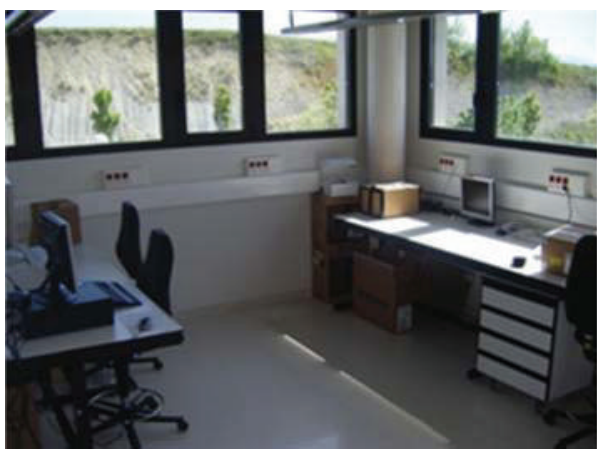

(a)

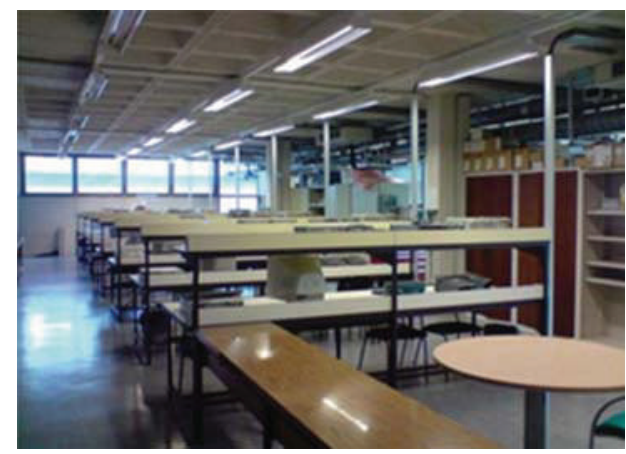

(b)

FIgURE 2: (a) R\&D building's laboratory no. 5 picture. (b) Radio communication laboratory picture.

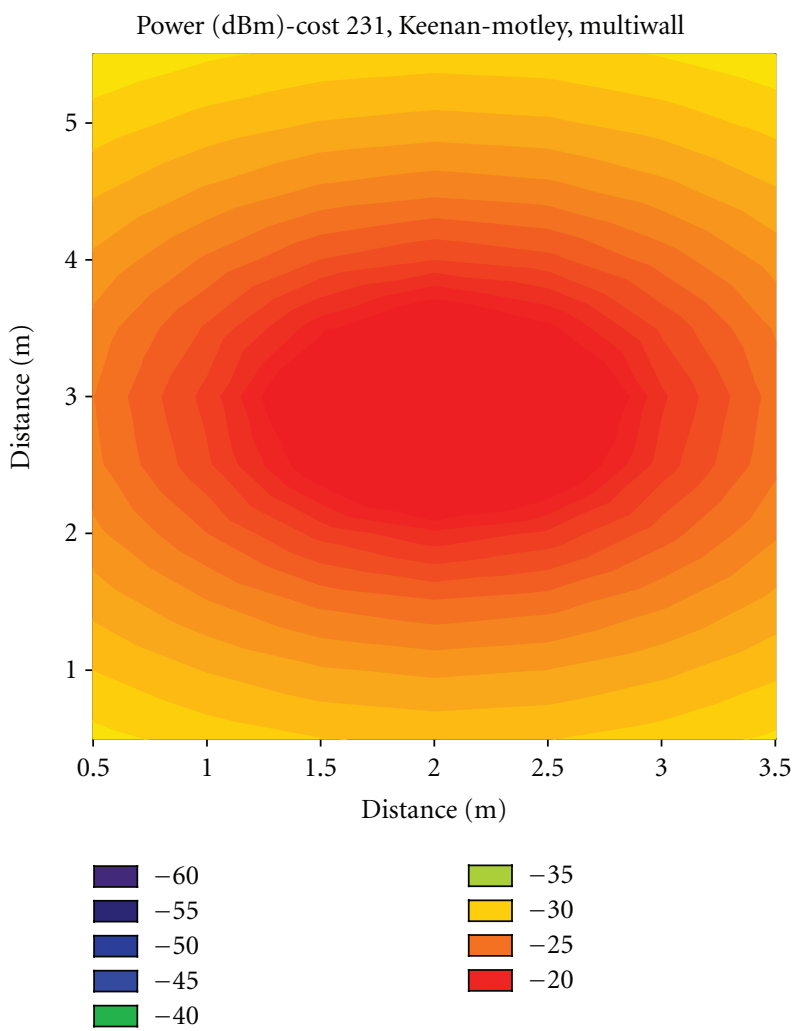

(a)

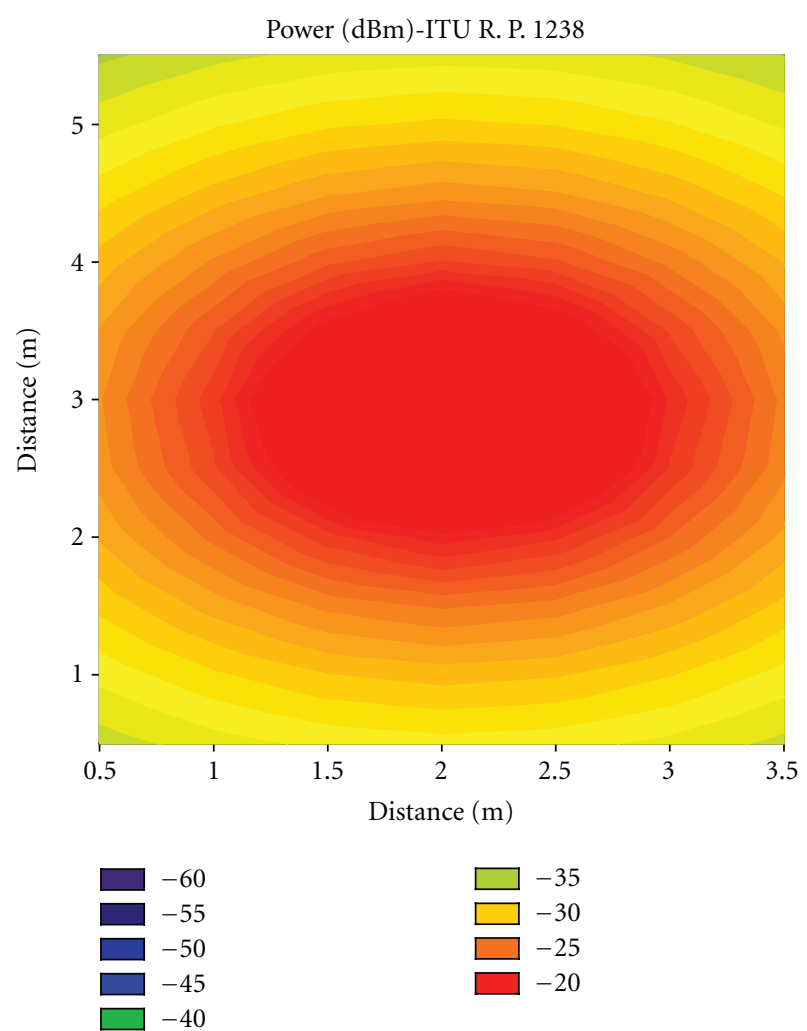

(b)

FIgURE 3: (a) Spatial distribution of received power $(\mathrm{dBm})$ for a height of $1.05 \mathrm{~m}$ in the indoor scenario of Figure 2(a) with Cost 231, Keenanmotley, and multiwall models. (b) Spatial distribution of received power ( $\mathrm{dBm}$ ) for a height of $1.05 \mathrm{~m}$ in the indoor scenario of Figure 2 (a) with ITU R. P. 1238 model. 


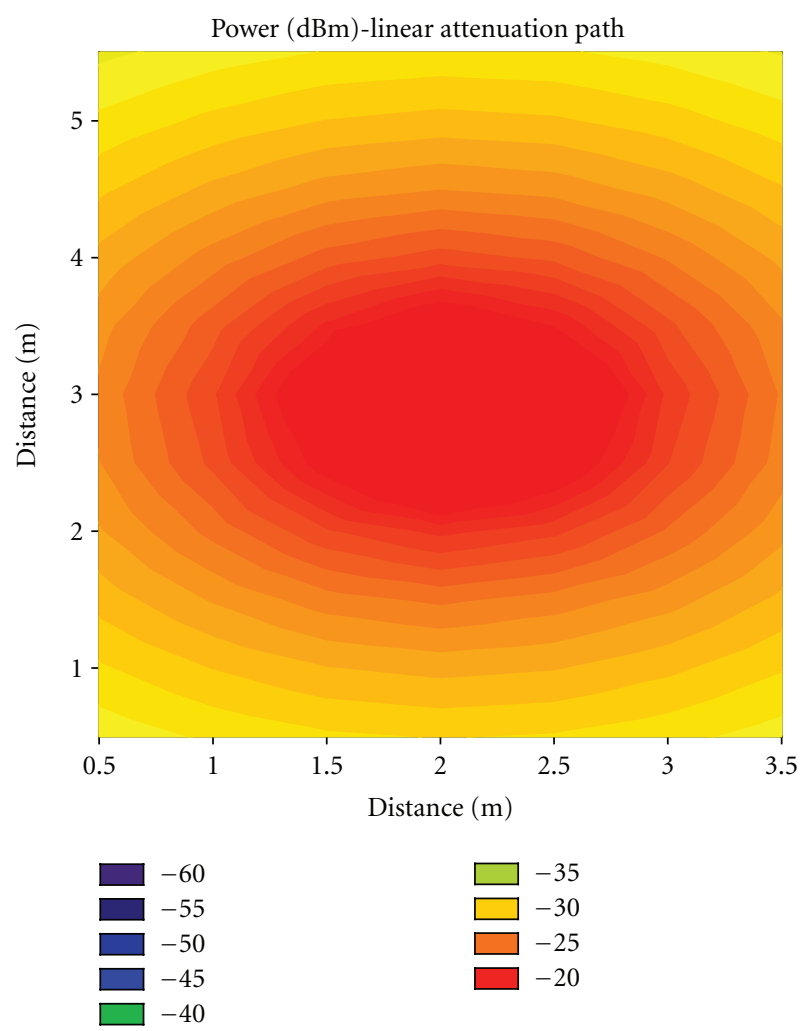

Figure 4: Spatial distribution of received power $(\mathrm{dBm})$ for a height of $1.05 \mathrm{~m}$ in the indoor scenario of Figure 2(a) with Linear Attenuation Path model.

between sensors and actuators governed by the intelligence of the system. Over time these systems have evolved incorporating more and more intelligence and decision making, up to ambient intelligence (AmI) concepts. Nowadays, AmI is described as an emerging discipline that brings intelligence to our everyday environments and makes them sensitive to end users. In order to obtain this level of intelligence, the hardware becomes more complex and is necessary to involve a larger number of sensors to obtain a large amount of information achieving a more accurate response. In this scenario, wireless sensors networks play a fundamental role, bringing the "Internet of Things" to the domestic environment, providing communications and intelligence to the devices that surround us in everyday life defining "new" ambient intelligence environments.

With the rapid development of the wireless network technologies, the Home Automation systems based on the WSN becomes more and more popular [27-39]. The main advantage of using these wireless sensors is that they are easy and fast to deploy, not requiring any kind of wiring infrastructure. There are many wireless communication technologies available (i.e., Bluetooth, ZigBee [40-42], Wi$\mathrm{Fi}$, NFC, etc.), most of them working in any of the existent ISM (Industrial, Scientific, and Medical) free bands. For Home Automation systems, aspects like low power consumption and low cost are typical parameters desirable in this kind of networks and because the data amount to exchange is small, the transmission data rate is not an important issue. There are several products in the market that fulfill these characteristics and most of them are IEEE 802.15.4 compliant devices and the most promising of them all. The main radio characteristics of the IEEE 802.15.4 protocol are shown in the Figure 1.

The goal of this work is to assess the radio coverage [43-45] and link quality [43] of IEEE 802.15.4 compliant devices in typical real indoor scenarios [46], for optimizing their deployment into WSN for using them on Home Automation systems. The proposed analysis is applicable in principle to a wide range of wireless systems, although it has been particularized for 802.15 .4 devices, which can be made extensible to ZigBee if higher layer functionalities are necessary. This type of wireless sensor network exhibit properties such as $5 \mathrm{MHz}$ bandwidth channelization, channel access based on CSMA/CA and low output power in the order of $0 \mathrm{dBm}$, just to name a few. These properties, together with multiple network configurations and the possibility of including a large number of nodes, pose 802.15.4 networks and ZigBee as candidates in home automation systems for control illumination, household appliance intercommunication, control of climate systems, presence-intrusion detection or home entertainment, among others. The main analysis parameter is link quality, which is given by the packet error ratio (PER), directly related to the signal to noise ratio to be achieved as a function of transceiver sensitivity. The signal to Noise ratio is strongly conditioned by the received power levels, which depend on the radiopropagation environment. Therefore, once the received signal levels are estimated, the link quality parameters can be inferred.

The behavior of the radio channel in indoor scenarios $[47,48]$ is not a trivial issue and heavily depends of the complexity of the environment. The appearance of degradation effects fundamentally due multipath components but also of phenomenon like reflection, refraction, diffraction, and scattering among others make the study of the associated radio channel a complex task [49]. Here it is where this paper focuses its efforts in order to optimize the wireless sensors deployment to achieve a more efficient network coverage and consumption. Usually, empirical based models and simplified deterministic methods are employed (i.e., COST 231, Walfish-Bertoni, Okumura Hata, etc.) [50]. These kinds of methods are fast and easy to calculate but not highly accurate. They require calibration based on measurements in order to give an adequate fit of the results, based on initial regression methods. On the other hand, deterministic methods are based on numerical approaches to the resolution of Maxwell's equations, such as ray launching and ray tracing (based on geometrical approximations) [51] or full wave simulation techniques (MoM, FDTD, FITD, etc.). These methods are precise, but are time consuming to inherent computational complexity. As a midpoint, methods based on geometrical optics, for radio planning calculations with strong diffractive elements, offer a reasonable tradeoff between precision and required calculation time [5254]. Simulations and measurements have been made in two laboratories of the Public University of Navarre: R\&D building's laboratory no. 5 (scenario 1) and the Radio 


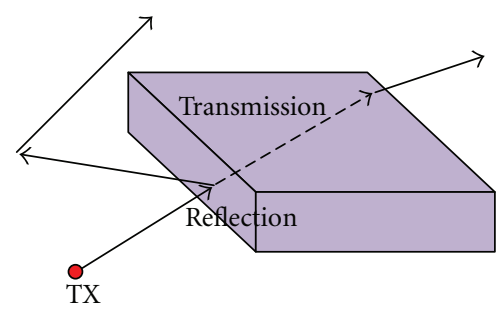

(a)

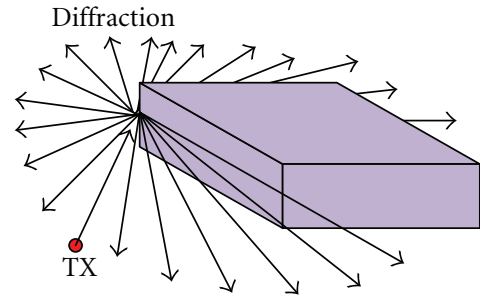

(b)

FIGURE 5: Principle of ray launching method: reflection, transmission, and diffraction.

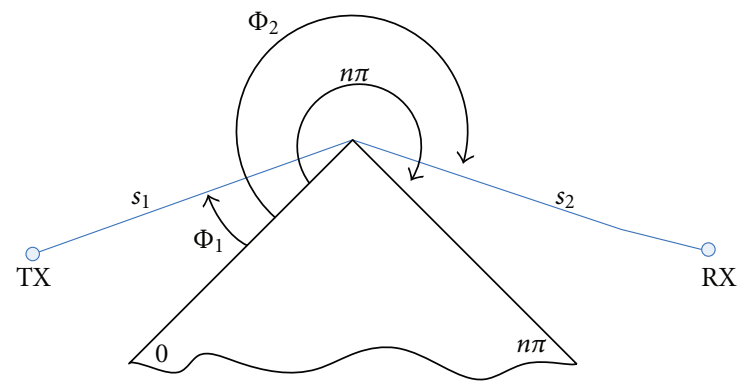

FIGURE 6: Geometry for wedge diffraction coefficients.

communication laboratory (scenario 2) depicted in the Figures 2(a) and 2(b).

These scenarios are complex environments composed of different types of walls (concrete, plywood, etc...) and a variety of different furniture (metallic cupboards, tables, chairs, computers, etc.) heavily affected by signal degradation due to multipath components. The proposed scenarios emulate the behavior of a typical small home's room and a more complex, bigger location like an office.

\section{Simulation Technique and Results}

In order to analyze the impact of the topology and morphology of the indoor scenario (i.e., domestic household, office building, etc.), in wireless system behavior, initial results can be obtained by simulation. A first approximation can be given by means of empirical based-models, based on analytical expressions derived from nonlinear regression of the scenario under analysis. These models give rapid results, but require calibration based on measurements in order to give an adequate fit of the result, since it is strongly dependent on the environment where the results are being obtained. In this work, the estimation of wireless coverage of scenario 1 has been obtained by applying different models, such as Cost 231, Keenan-Motley, and multiwall models (see Figure 3(a)), ITU R. P. 1238 model (see Figure 3(b)) and linear attenuation path model (see Figure 4 ). The calculations have been obtained considering a frequency of operation of $2.45 \mathrm{GHz}$ and a transmitter power of $18 \mathrm{dBm}$. As it can be seen from the figures, these models give simplified estimations, in which the complexity of the indoor elements (such as material changes, furniture, windows, etc.) has no significant effect.

In addition to this, a deterministic method based on $3 \mathrm{D}$ ray launching is used in order to analyze the indoor scenario. A 3D ray launching algorithm has been implemented inhouse, based on MatLab programming environment. It is based on geometrical optics (GO) and geometrical theory of diffraction (GTD). The rays considered in GO are only direct, reflected, and refracted rays. Because of this, abrupt transitions areas may occur, corresponding to the boundaries of the regions where these rays exist. To complement the GO theory, the diffracted rays are introduced with the GTD and its uniform extension, the Uniform GTD (UTD). The purpose of these rays is to remove the field discontinuities and to introduce proper field corrections, especially in the zero-field regions predicted by GO.

The basic procedure of the ray launching algorithm is, first, to launch a ray from the transmitting antenna (Tx). Then, the ray is traced to see if it hits any object or is received by the receiving antenna. When the ray impacts with an obstacle, reflection, transmission, and diffraction will occur, depending on the geometry and the electric properties of the object. Figure 5 represents the phenomena of reflection and transmission when the ray impacts with an obstacle and the diffraction phenomena when the ray impacts with an edge.

A plane electromagnetic wave falling to the planar interface between two regular semi-infinite media 1 and 2 gives rise to two plane waves: reflected and transmitted (or refracted). According to the Snell's law [55], the reflection coefficient $R^{\perp}$ and transmission coefficient $T^{\perp}$ are calculated by

$$
\begin{aligned}
T^{\perp} & =\frac{E_{t}^{\perp}}{E_{i}^{\perp}}=\frac{2 \eta_{2} \cos \left(\Psi_{i}\right)}{\eta_{2} \cos \left(\Psi_{i}\right)+\eta_{1} \cos \left(\Psi_{t}\right)}, \\
R^{\perp} & =\frac{E_{r}^{\perp}}{E_{i}^{\perp}}=\frac{\eta_{2} \cos \left(\Psi_{i}\right)-\eta_{1} \cos \left(\Psi_{t}\right)}{\eta_{2} \cos \left(\Psi_{i}\right)+\eta_{1} \cos \left(\Psi_{t}\right)},
\end{aligned}
$$

where $\eta_{1}=120 \pi / \sqrt{\varepsilon_{r 1}}, \eta_{2}=120 \pi / \sqrt{\varepsilon_{r 2}}$, and $\Psi_{i}, \Psi_{r}$, and $\Psi_{t}$ are the incident, reflected, and transmitted angles respectively.

For the parallel (or magnetic) polarization the magnetic field vector of the incident wave is perpendicular to the 


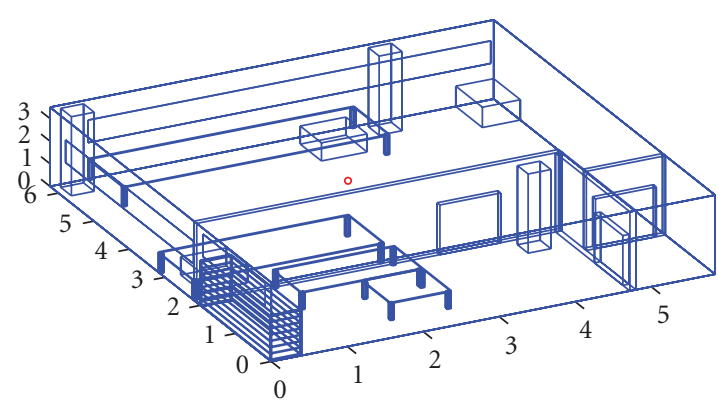

(a)

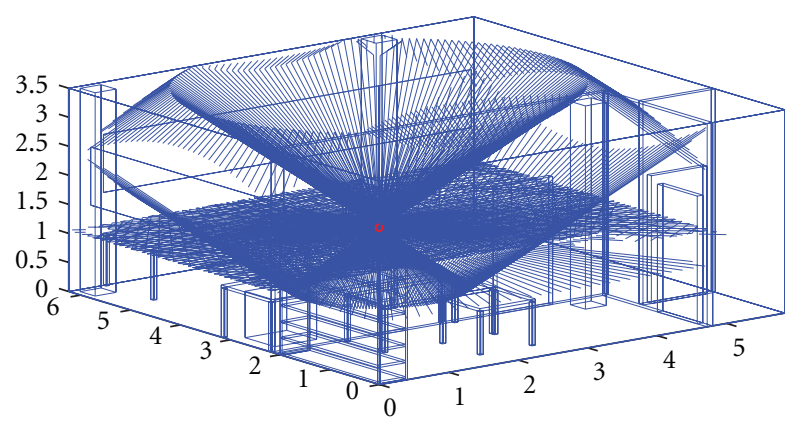

(b)

FIGURE 7: (a) R\&D building's laboratory no. 5, proposed for deterministic radio channel simulation. (b) Ray launching representation for the indoor scenario. Rays are launched given the solid angle definition within the simulation parameters.
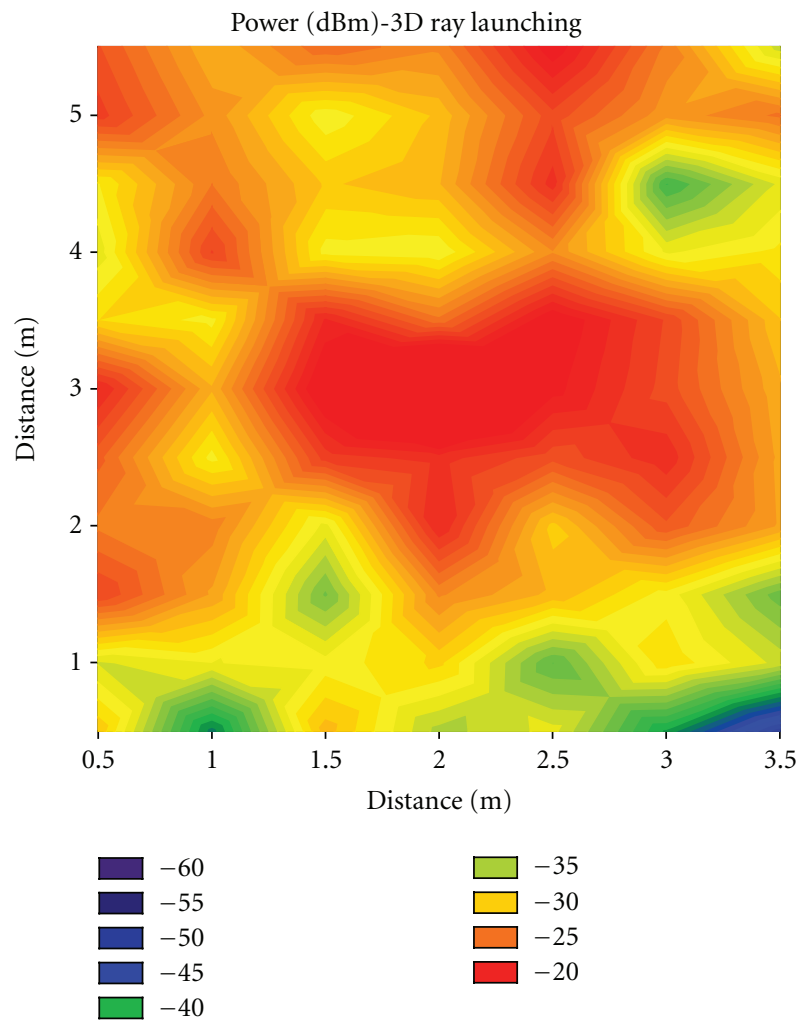

FIGURE 8: Bidimensional distribution of received power $(\mathrm{dBm})$ for a height plane of $1.05 \mathrm{~m}$ in the indoor scenario of Figure 2(a) calculated with the $3 \mathrm{D}$ ray launching algorithm.

plane of incidence. Then, the reflection and transmission coefficients $R^{\|}$and $T^{\|}$can be calculated by

$$
\begin{aligned}
& R^{\|}=\frac{E_{r}^{\|}}{E_{i}^{\|}}=\frac{\eta_{1} \cos \left(\Psi_{i}\right)-\eta_{2} \cos \left(\Psi_{t}\right)}{\eta_{1} \cos \left(\Psi_{i}\right)+\eta_{2} \cos \left(\Psi_{t}\right)}, \\
& T^{\|}=\frac{E_{t}^{\|}}{E_{i}^{\|}}=\frac{2 \eta_{2} \cos \left(\Psi_{i}\right)}{\eta_{1} \cos \left(\Psi_{i}\right)+\eta_{2} \cos \left(\Psi_{t}\right)} .
\end{aligned}
$$

Once the parameters of transmission $T$ and reflection $R$ are calculated, and the angle of incidence $\Psi_{i}$ and $\Psi_{t}$, the

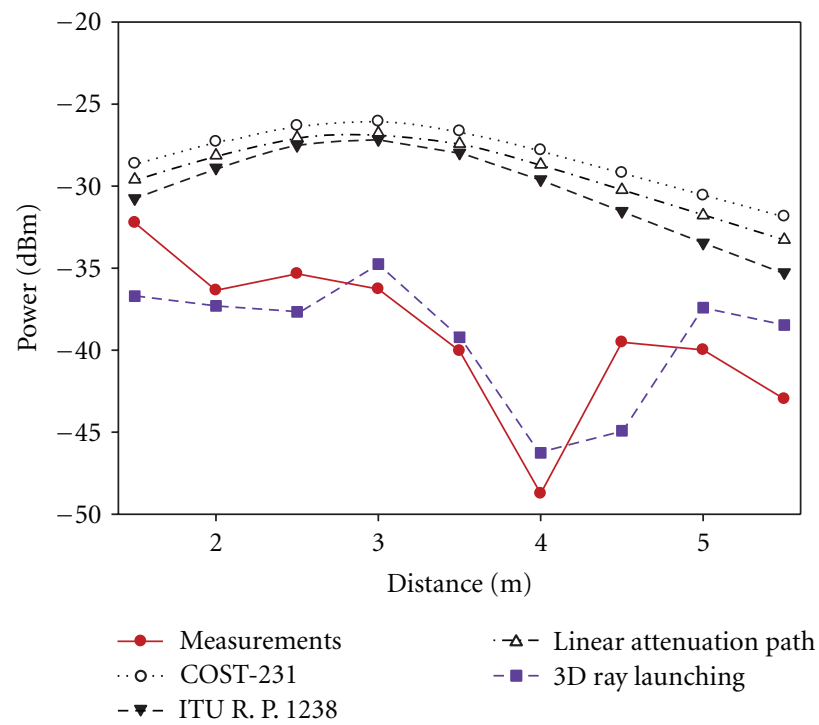

FIgure 9: Comparison Measurements versus different Propagation Models for $X=0.5 \mathrm{~m}$ along the $Y$-axis.

new angles $\left(\theta_{r}, \phi_{r}\right)$ of the reflected wave, and $\left(\theta_{t}, \phi_{t}\right)$ of the transmitted wave can be calculated.

The finite conductivity two-dimensional diffraction coefficients are given by [56-58] as follows:

$$
D^{\| \perp}=\frac{-e^{(-j \pi / 4)}}{2 n \sqrt{2 \pi k}}
$$

$$
\times\left\{\begin{array}{l}
\cot g\left(\frac{\pi+\left(\Phi_{2}-\Phi_{1}\right)}{2 n}\right) F\left(k L a^{+}\left(\Phi_{2}-\Phi_{1}\right)\right) \\
+\cot g\left(\frac{\pi-\left(\Phi_{2}-\Phi_{1}\right)}{2 n}\right) F\left(k L a^{-}\left(\Phi_{2}-\Phi_{1}\right)\right) \\
+R_{0}^{\| \perp} \cot g\left(\frac{\pi-\left(\Phi_{2}+\Phi_{1}\right)}{2 n}\right) F\left(k L a^{-}\left(\Phi_{2}+\Phi_{1}\right)\right) \\
+R_{n}^{\| \perp} \cot g\left(\frac{\pi+\left(\Phi_{2}+\Phi_{1}\right)}{2 n}\right) F\left(k L a^{+}\left(\Phi_{2}+\Phi_{1}\right)\right)
\end{array}\right\},
$$




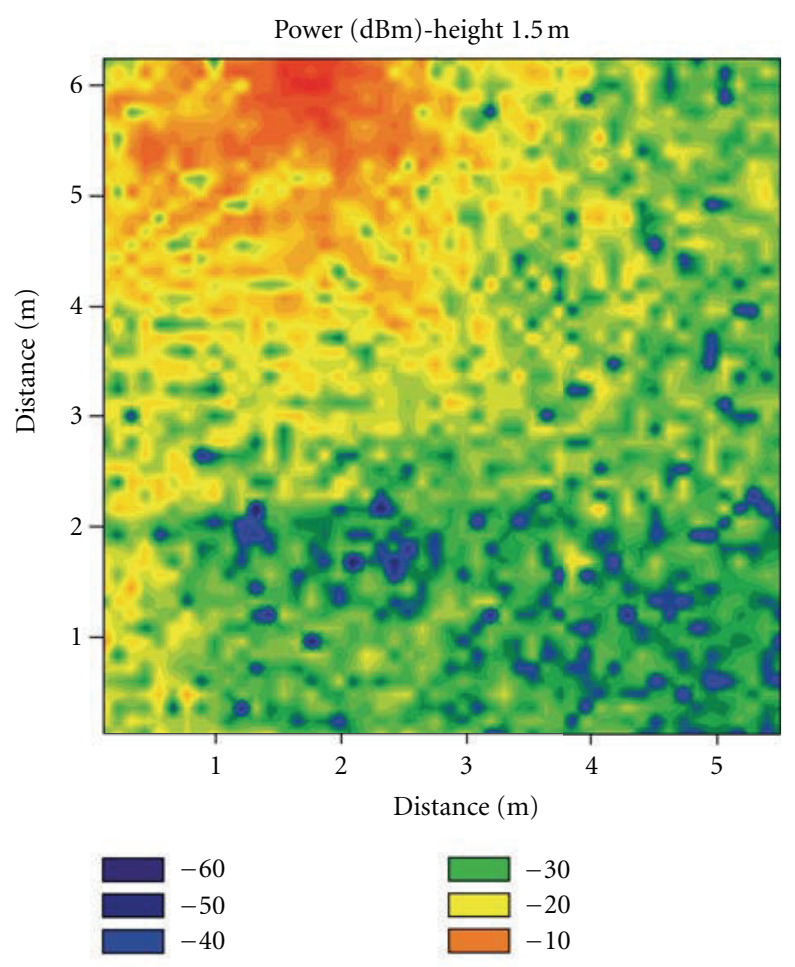

(a)

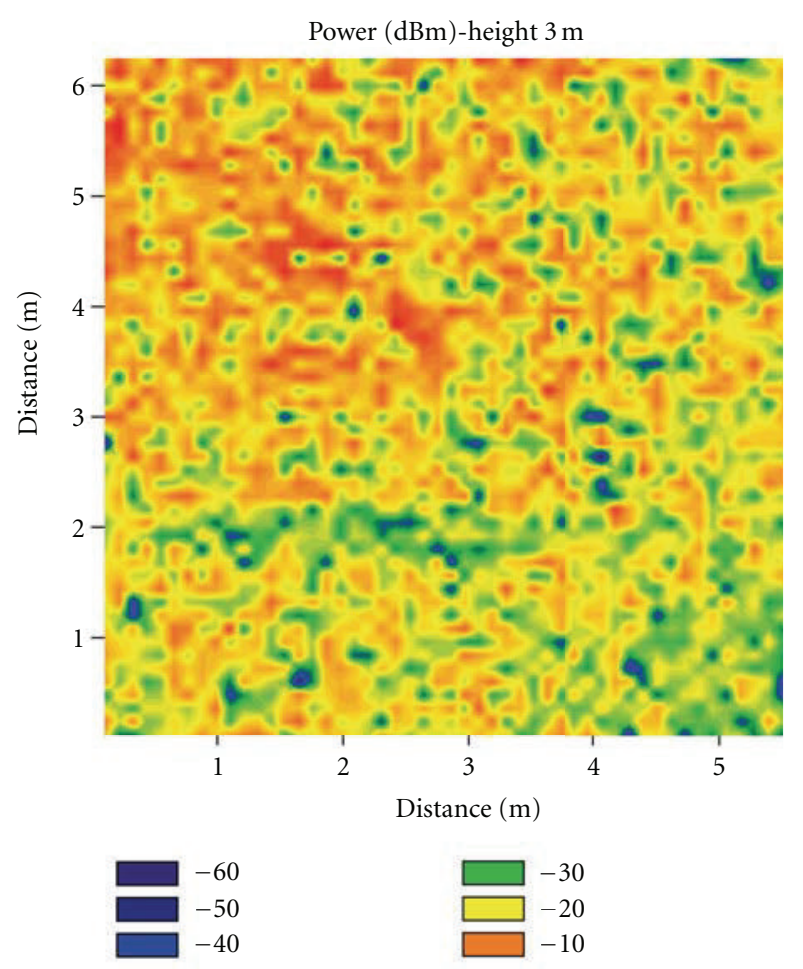

(b)

FiguRe 10: Spatial distribution of received power $(\mathrm{dBm})$ for different heights in the indoor scenario of Figure 2(a) obtained by means of 3D ray launching algorithm.

where $n \pi$ is the wedge angle, $F, L$, and $a \pm$ are defined in [51], $R_{0, n}$ are the reflection coefficients for the appropriate polarization for the 0 face or $\mathrm{n}$ face, respectively. The $\Phi_{2}$ and $\Phi_{1}$ angles in (3) would refer to the angles in Figure 6.

Within the considered indoor scenario, several sources (which can emulate sensors connected by wireless technology, WLAN or femtocells, as part of an Intelligent Home or Automated Building) can be placed, in which wireless power is converted into a finite number of rays launched within a solid angle. Parameters such as frequency of operation, radiation diagram of the antennas, number of reflections, separation angle between rays, and cuboid dimension can be fixed.

A view of the indoor scenario (which correspond to the "R\&D building's laboratory No. 5" shown in Figure 2(a), including indoor elements such as furniture, doors and walls) is shown in Figure 7(a). In Figure 7(b), a representation of the ray launching procedure is shown, with a three dimensional calculation scheme. The scenario is subdivided in cuboids, in which the calculation of electrical field values in vector format are performed and processed in order to estimate parameters such as received power levels or power delay profiles, as a function of the spatial observation point.

In order to analyze the influence of the topology and morphology of the wireless network, the indoor simulation scenario depicted in Figure 2(a) has been simulated. Figure 8 shows the simulation results in the same points of the measurements. It is observed that results are more accurate with deterministic model than empiric ones, given by the consideration of the elements within the indoor scenario. The simulations have been performed considering a frequency of operation of $2.45 \mathrm{GHz}$ and a transmit power equal to $18 \mathrm{dBm}$.

Figure 9 shows the comparison between real measurements, empiric models and deterministic model. As it can be seen, there is good agreement between measurement results and $3 \mathrm{D}$ ray launching results, whereas there is worse agreement with empirical based models in general. The illustration correspond to the values of $X=0.5$ along the $Y$-axis, with higher discrepancies at certain points, due to averaging differences in the cuboid and equivalent antenna volume. The adequate correlation between simulation and measurement results validates the simulation results for the complete scenario. As stated previously, the determination of the received signal levels is the key parameter in order to analyze the overall link quality of the wireless sensor network nodes.

Several simulations have been performed in different locations of the transmitter to verify that the interaction with the elements within the indoor scenario are considered and to stress the fact that different material properties play a key role in the overall performance of the wireless system.

Figure 10 shows the received power for two different heights in the indoor scenario with the transmitter fixed at the point $(1.8,5.34,1.05)$ meters. Once the values of received power can be estimated within the scenario, information 

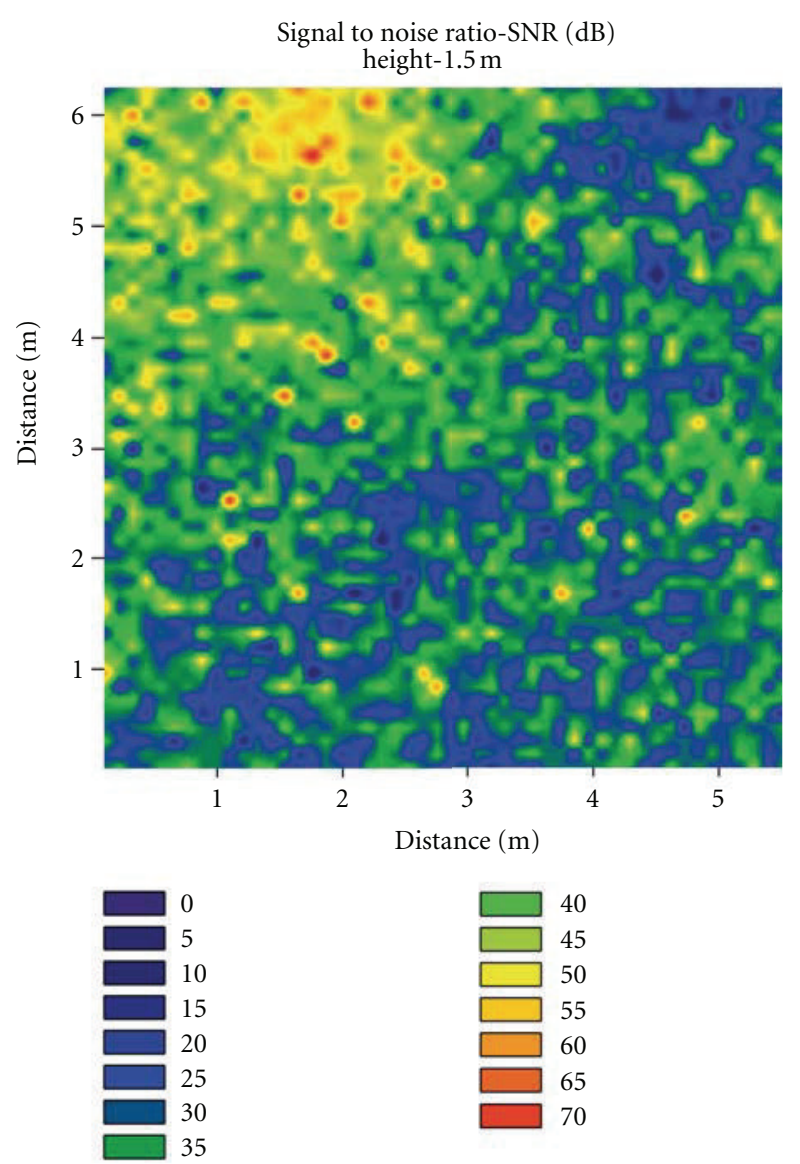

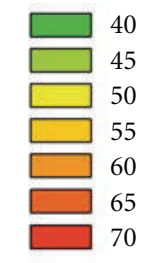

(a)
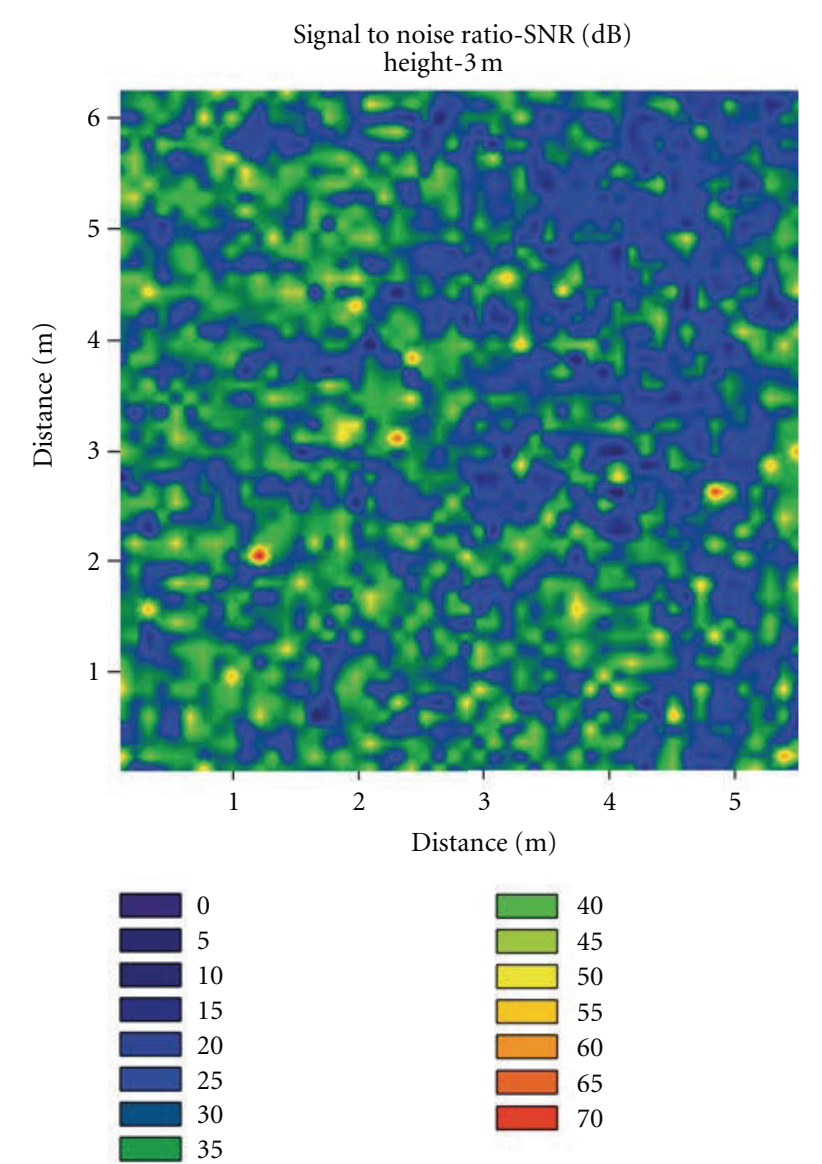

(b)

FIGURE 11: Spatial distribution of signal to noise ratio for different heights in the indoor scenario of Figure 2(a) with the 3D ray launching algorithm.

related to the performance of wireless systems can be analyzed. As an example, Figure 11 represents the signal to noise ratio for the same heights in the scenario as in the previous case. This information can be used in order to consider the most adequate deployment strategy of a set of wireless sensor networks within the indoor scenario, in order to increase signal quality, whilst maintaining the optimal wireless power transmission (and hence energy consumption) of the system.

As it can be seen, there is noticeable variation even in the case of relatively small distances. This is due to the particular electromagnetic phenomena occurring within the scenario, such as fast fading, which is the most relevant, that is due to the multipath components, strongly dependent of the morphology and topology of the scenario considered. As for consideration of computational error, the corresponding simulation error is defined as the difference between the measured and simulated values of the path loss at any time. The resulting mean standard error is 0.501 and the standard deviation 1.585 , indicating that the simulation method is working properly. It is also worth noting that the simulation results indicate that the received signal levels are above the sensitivity threshold of conventional 802.15.4 transceivers, usually below $-80 \mathrm{dBm}$ and dependent on the signal to noise ratio within the region of operation.

Additional results of signal to noise ratio for different height planes are shown in Figure 11, which confirm the variation as a function of the position of the receiver within the bidimensional plane. Once again, this is a consequence of the changes in the distribution of materials and elements within the indoor scenario, which is responsible for the components of multipath propagation, which is very significant in this type of scenarios. To illustrate the relevance of this propagation mechanism the power delay profile for a given point of the scenario is shown in Figure 12. As it can be seen, there is a large number of echoes in the scenario, within a time span of approximately $15 \mathrm{~ns}$ to $90 \mathrm{~ns}$, corresponding to distances from 0.5 meters to 2.5 meters. The large amount of echoes within such a short distance is coherent with the complexity of the scenario (elements such as furniture) as well as the material properties at the frequency of operation.

In order to illustrate the dependence with spatial distribution, Figure 13 represents how the received power is distributed along the $X$ - and $Y$-axis for different heights. The strong dips in received power level are due to destructive 


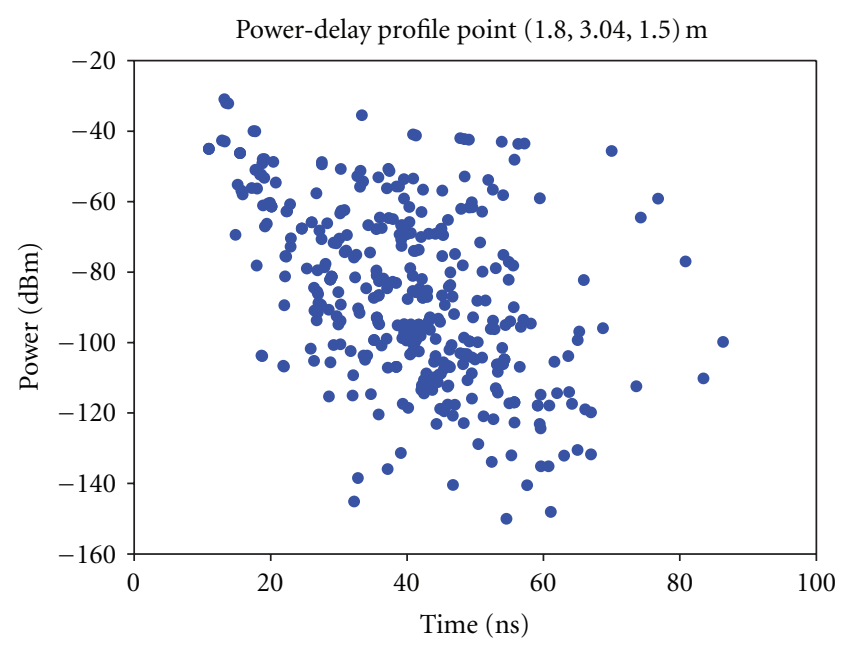

Figure 12: Power-delay profile at point $(1.8,3.04,1.5) \mathrm{m}$ in the indoor scenario.

addition of multipath components, described by statistically by fast fading. This information is useful in the estimation of optimal position of sensor elements within the scenario, in order to obtain the highest possible reception value by carefully analyzing the deployment strategy.

In addition, several simulations of the indoor scenario "radio communication laboratory" (Figure 2(b)) have been done. Figure 14 shows the spatial distribution of power for different heights in this scenario with the antenna fixed at the point $(2,2$, height $=0.81) \mathrm{m}$. In this case, the scenario has a larger size, with a great deal of furniture, typical of laboratory/technical office environment. Once again, the morphology and the topology of the scenario play a key role in the values of received power levels and hence in the overall sensor network performance.

\section{Experimental Setup}

The simulation results obtained in the previous section shows the relevance of the layout of the working scenario in the deployment of wireless sensor networks. Validation is now sought in terms of experimental results, with the aid of realistic wireless transceivers in different scenarios. Due to the performance in terms of unit cost, power consumption and spectrum availability, IEEE 802.15.4 based devices will be employed for the experimental setup.

The wireless communication devices used in this work are XBee Pro models, from Digi International Inc, shown in the Figure 15(a). The transmission RF power level of the wireless communication devices can be adjusted with a maximum default value of $18 \mathrm{dBm}$ which will be used in the experimental validation. The module has different antenna options (i.e., external antenna, usually a whip antenna, or a compact integrated chip antenna) with election, in terms of size and integration of chip antenna, which leads to a low antenna gain, in the order of $-1.5 \mathrm{dBi}$. For simple standalone operation, the transmitter XBee Pro module and the associated components can be plugged into a solderless breadboard powered by two 1.5 VDC AA batteries. For transmitting arbitrary data or processing receiving data, the connection with a PC is required. For that purpose, the XBee Pro module is plugged into an XBee Explorer USB unit (see Figure 15(b)). In this way, the XBee Pro modules UART appears as a USB serial port in the connected PC and can be accessed for reading/writing data using RS 232 protocol.

The initial experimental setup was deployed in a small office, located in an R\&D facility at the Public University of Navarre. In order to analyze the radio propagation characteristics of the scenario (see Figures 2(a) and 2(b)), a transmitting XBee Pro module was placed in the center of the room and the received RF power were registered in several different points. Received power values were measured with the aid of an Agilent N9912A FieldFox RF Spectrum Analyzer of Agilent with an omnidirectional antenna, with a central detection frequency fixed at $2.4 \mathrm{GHz}$. The spectrum analyzer is capable of measure signals from $5 \mathrm{KHz}$ to $4 / 6 \mathrm{GHz}$ with a DALN (displayed average noise level) of $-148 \mathrm{dBm}$, a sufficient dynamic range to analyze the XBee Pro transmitted $\mathrm{RF}$ signal. In order to improve the spectrum analyzer's measurement time, the transmitter was configured to operate sending one packet of data per millisecond, which is the maximum achievable data rate. The obtained measured RF power for each of the different points is represented in the Figure 16.

If we compare these measured values with the previous simulations results, it can be seen that the spatial distribution of the received RF power differs greatly from the values predicted by the propagation models and adjusts more precisely to the ones predicted by the $3 \mathrm{D}$ ray launching algorithm. Another important aspect of a wireless communication system is the quality of the radio link. It is significant to determine how the received packet's RSSI affects the links PER (packet error rate), as already stated previously. To obtain the data, XBee Pro module's functionality of estimating each received packet's RSSI was used. The goal is to analyze the relation of PER of the radio link according to different values of RSSI, since this will give a direct correlation between both values and hence allow an estimation of the overall observable radio link quality.

In one particular point of the room, an XBee Pro module emulating a transmitter was placed and connected to a computer via a USB cable. In the same computer, a Java application was placed running, which was programmed in-house for such purpose which broadcast a quantity of packets determined by the user. On the other hand, another XBee Pro module was connected, also via a USB cable, to a laptop. In that laptop another in-house Java programmed application was running. That application determines the average RSSI of the received packets and the corresponding PER value, also storing them in one text file together with the values of the arriving time, RSSI value and the sequence number of every received packet. The receiver's position was changed for obtaining different values of average RSSI and its corresponding PER value in different points. To obtain valid data, it is important to send a significant amount of packets. Taking this into consideration, the number of packets used for every PER measurement was of 1,000,000. 


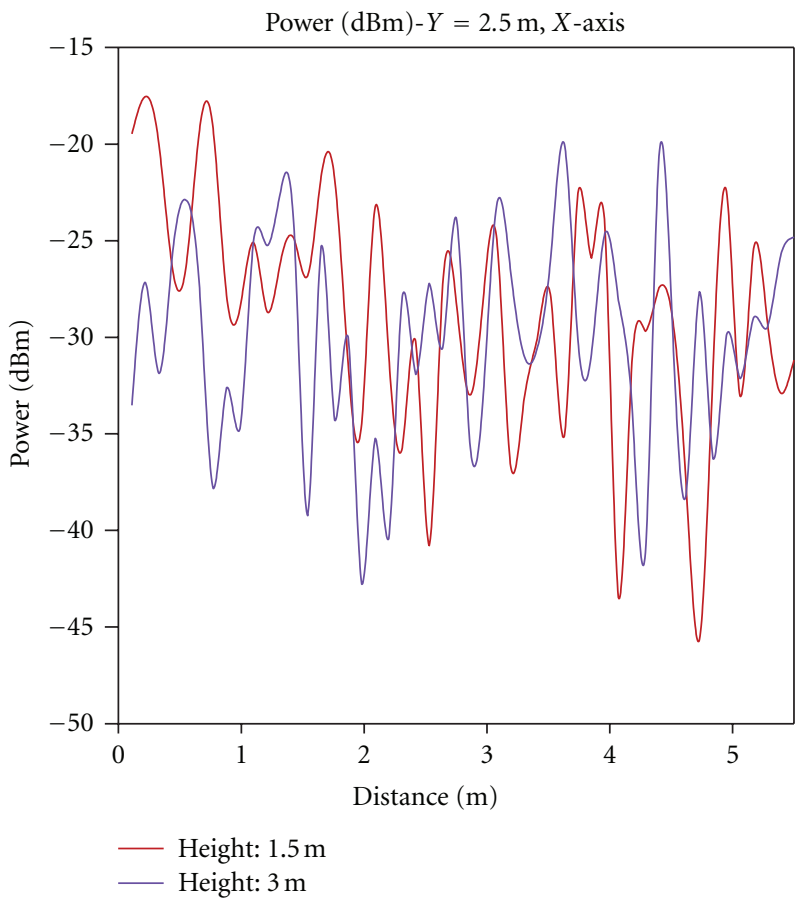

(a)

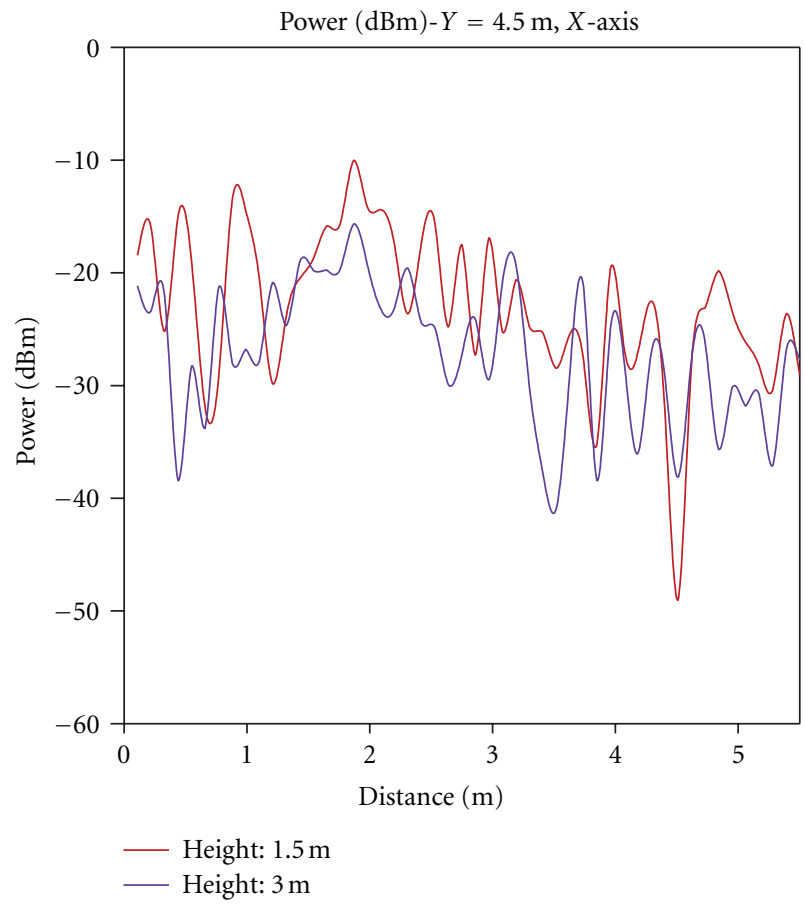

(b)

FIGURE 13: Distribution of power for different points of $Y$ along the $X$-axis and for different heights.

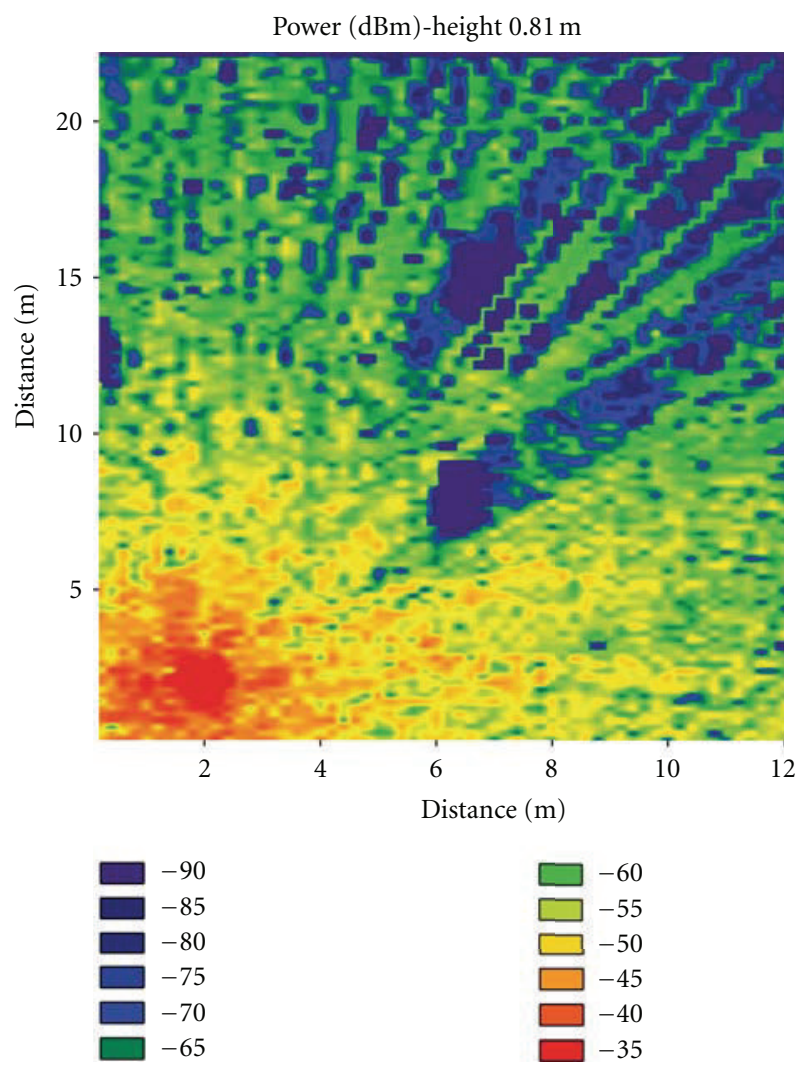

(a)
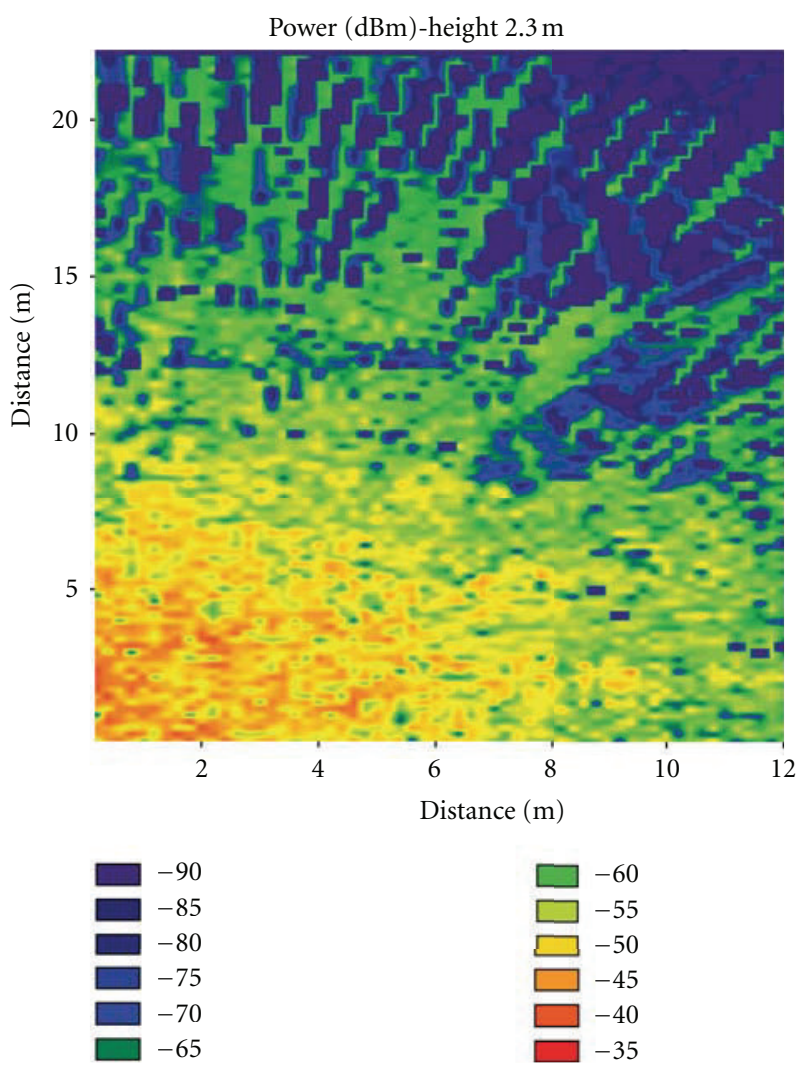

(b)

FigURE 14: Spatial distribution of received power ( $\mathrm{dBm}$ ) for different heights in the indoor scenario of Figure 2(b) with the 3D ray launching algorithm. 


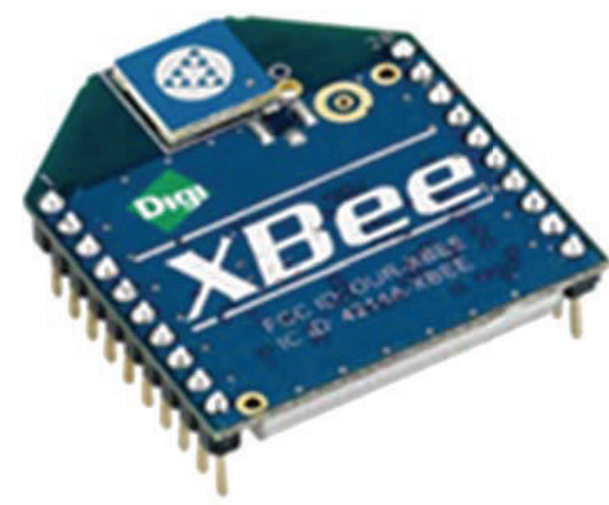

(a)

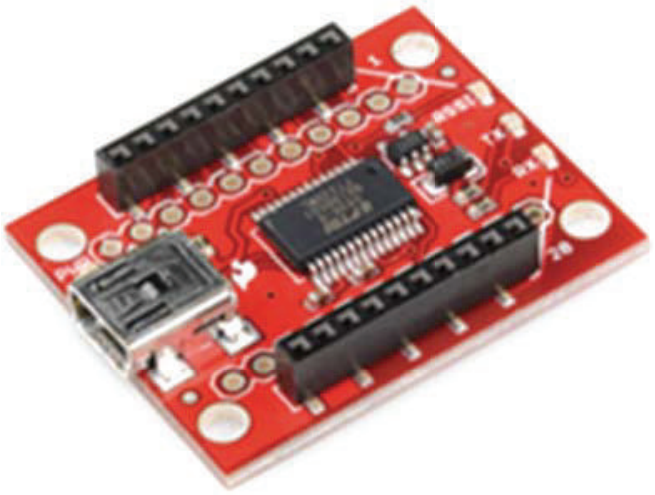

(b)

Figure 15: (a) XBee Pro module. (b) XBee Explorer USB.

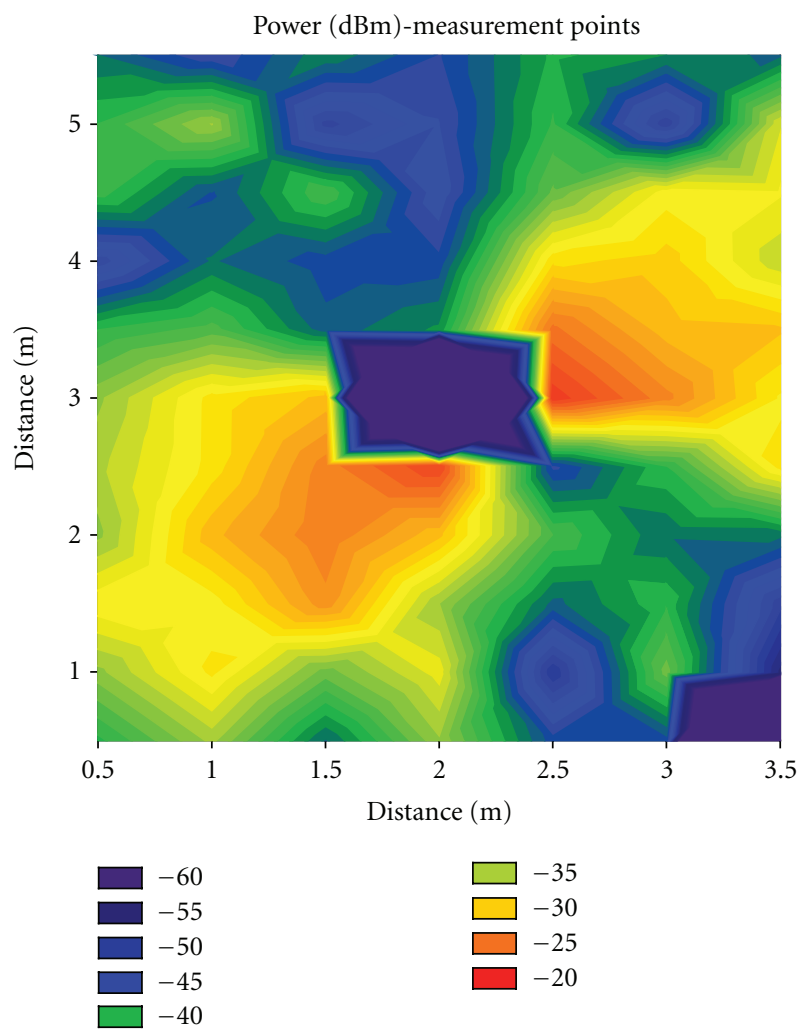

FIGURE 16: Measured RF power values for indoor scenario 1.

In Figure 17 the representation of the PER versus the average RSSI is shown. It can be seen that the behavior follows an exponential trend of received RF signal power and around $-85 \mathrm{dBm}$ the degradation of the signal is relevant. The IEEE 801.15.4 uses the DSSS (direct-sequence spread spectrum) modulation, which relies on spread spectrum for becoming more robust versus the radio channel noise, in a similar manner as an error-correction code. For every data symbol of 4 bits, a 32 bit chip symbol is send and the receiver can retrieve the original signal even with noise presence. This issue is very important due the quantity of different noise

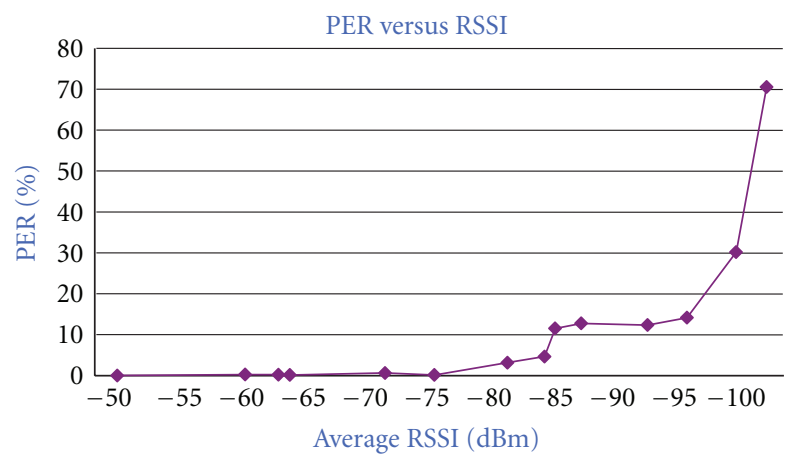

FIGURE 17: Representation of PER versus average RSSI in scenario 1.

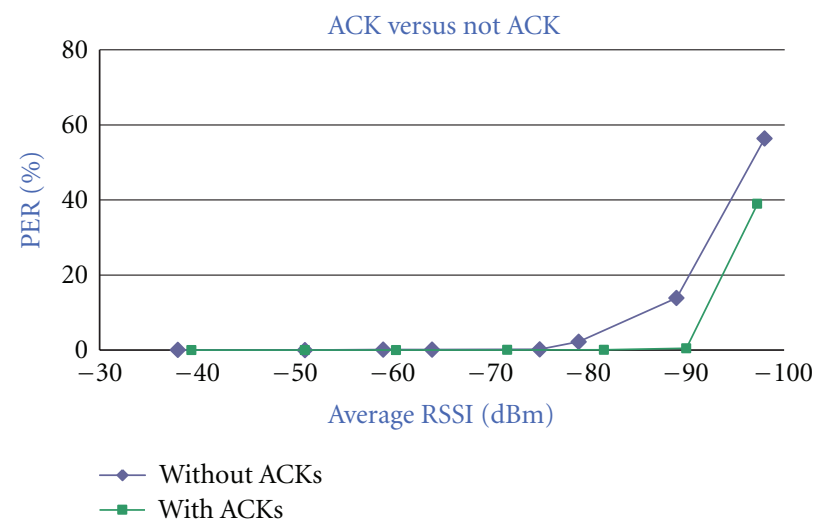

FIGURE 18: Representation of wireless link degradation in terms of percentage of PER, with or without use of ACK.

interferences that can be present in the radio channel, like microwave ovens, Bluetooth devices, Wi-Fi devices, and so forth.

When the signal to noise ratio becomes too low, the receiver begins to have problems retrieving the original data correctly. This effect is what we see in Figure 17.

The XBee Pro module, in its network layer, has the capability of sending ACK (acknowledgment) packets for 


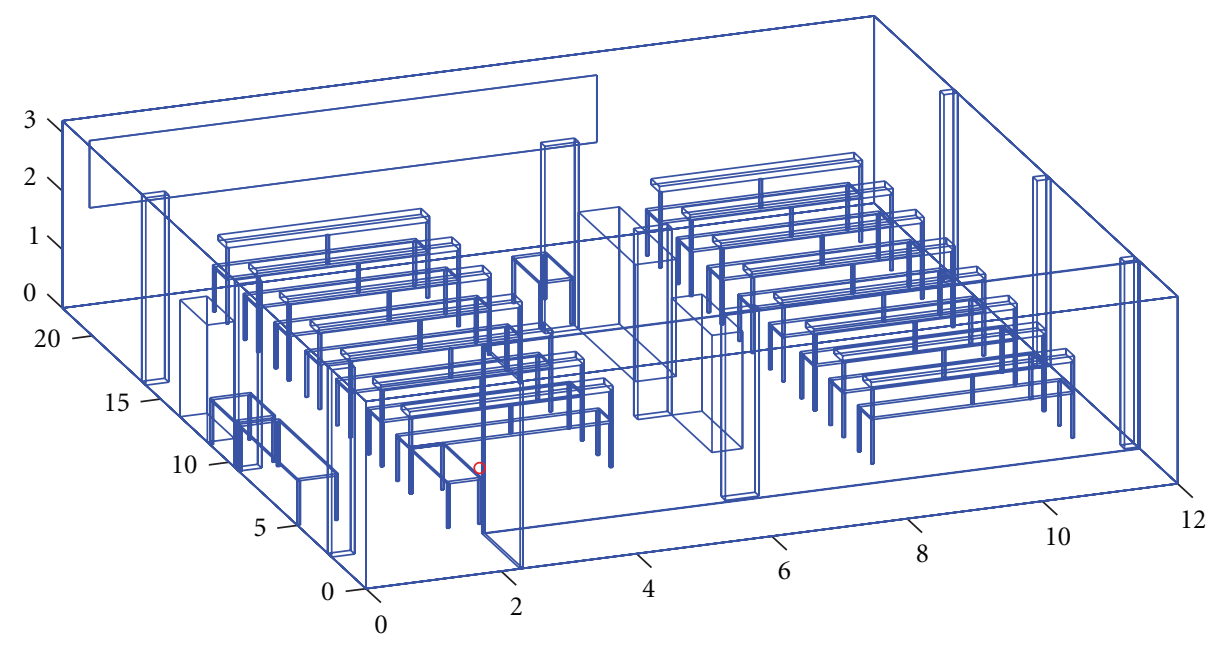

FIgURE 19: Scenario 2: Radio communication laboratory.

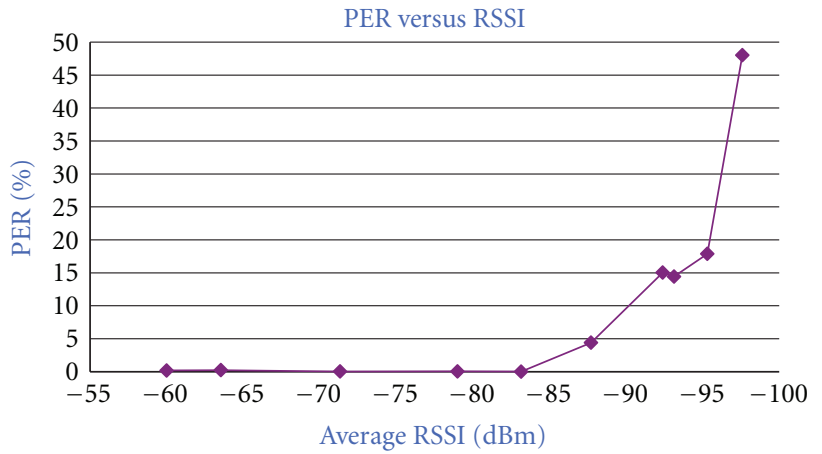

FIGURE 20: Representation of PER versus average RSSI in scenario 2.

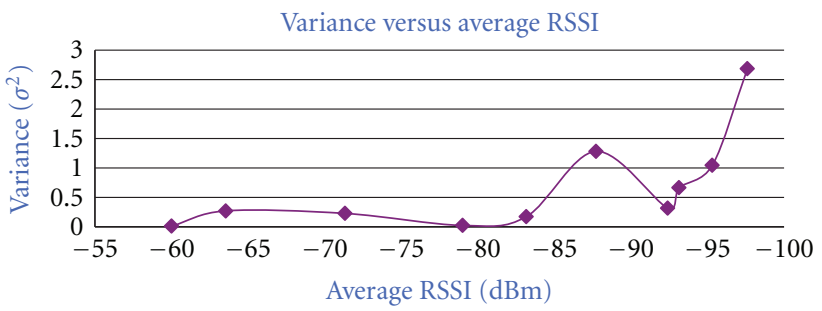

FIGURE 21: Standard deviation curve for the measurement results previously obtained.

error recovering. When a packet is sent by the transmitter, a ACKs retry packet is sent back from receiver. If a packet is sent with no ACK retry, another packet is sent. The IEEE 802.15.4 protocol provides a maximum of 3 ACK retries after the packet emission is considered a failure. For the purpose of this analysis, this functionality will been deactivated in all measurements.

The difference in the link quality between using or not an ACK could be very significant, as it is shown in Figure 18. It can be seen that the trend of both curves is similar; however, the degradation in data quality (given by the percentage of errors in the packets, PER) is observed with received power levels that exhibit a difference of over $20 \mathrm{~dB}$, which has a strong impact on the overall coverage radius of the wireless system. These data values have been measured in the same scenario, transmitting 500,000 data packets.

Further results have been obtained for a more complex scenario, depicted in a schematic way in Figure 19. In this case, the indoor scenario corresponds to a real laboratory, with a higher density of elements, such as different type of furniture and a larger surface.

The obtained measurement data of a deployed wireless sensor topology within the scenario is shown in Figure 20, as a representation of the PER versus the average RSSI. As it is expected, similar behavior is observed as in the previous case of scenario 1. In this case, degradation in the amount of received packets is observed at a level of approximately $-82 \mathrm{dBm}$, lower than in the precious case. This is due to the fact that the complexity of the scenario increases the amount of radio propagation losses, which leads to more conservative network deployment of the wireless sensors. To obtain the average RSSI value for every measurement point, the corresponding variance has been calculated also. In Figure 21, the representation of the standard deviation values versus the average RSSI values is shown, indicating a stable measurement condition all over the points within the scenario.

\section{Conclusions}

The layout and composition of elements within an indoor scenario play a key role in the overall effect in terms of losses in the propagation channel used in wireless sensor networks and systems. Initial considerations are based on simplified empirical based models, which lead to large errors in received signal estimation. To overcome this limitation, an in-house deterministic code based on 3D ray launching has been developed to analyze the effect of the indoor topology and morphology in the operation of wireless links within two different realistic scenarios. Experimental validation has 
been obtained by performing tests with test RF signals as well as with wireless sensors, which have been specifically programmed in order to obtain performance parameters, such as RSSI and PER indicators. The results confirm that the analysis of the topology of the wireless sensor network has a strong impact in complex indoor scenarios, in which small distances represent relevant changes in the received level as well as the overall quality of the wireless system. The application of deterministic techniques in the planning phase of wireless sensor networks and systems has a direct impact in the quality of service, capacity and overall energy consumption, which is of great importance in the deployment strategy of future high density sensor configurations, present in home automation as well as building automation systems of the future.

\section{Acknowledgments}

This work has been supported by the following Projects ref. IIM010566 and RI1 from the Government of Navarre, Industry Department and by the Spanish "Ministerio de Educación y Ciencia" through Project TEC2007-67987-C0202 .

\section{References}

[1] R. Bose, "Sensor networks-motes, smart spaces, and beyond," IEEE Pervasive Computing, vol. 8, no. 3, pp. 84-90, 2009.

[2] W. Dargie and C. PoellaBauer, Fundamentals of Wireless Sensor Networks Theory and Practice, John Wiley \& Sons, Chichester, UK, 2010.

[3] Y. Xiao and Y. Pan, Emerging Wireless LANs, Wireless PANs, And Wireless MANs, John Wiley \& Sons, Hoboken, NJ, USA, 2009.

[4] S. A. Mitilineos, D. M. Kyriazanos, O. E. Segou, J. N. Goufas, and S. C. A. Thomopoulos, "Indoor localization with wireless sensor networks," Progress in Electromagnetics Research, vol. 109, pp. 441-474, 2010.

[5] J. H. Sharly, T. Y. Choi, J. H. Park, S. H. Kang, S. J. Yun, and J. G. Park, "On-line ranging for mobile objects using ZIGBEE RSSI measurement," in Proceedings of the 3rd International Conference on Pervasive Computing and Applications (ICPCA '08), pp. 662-666, Alexandria, Egypt, October 2008.

[6] L. Ruiz-Garcia, P. Barreiro, J. I. Robla, and L. Lunadei, "Testing zigBee motes for monitoring refrigerated vegetable transportation under real conditions," Sensors, vol. 10, no. 5, pp. 4968-4982, 2010.

[7] J. A. Gay-Fernández, M. G. Sánchez, I. Cuiñas, A. V. Alejos, J. G. Sánchez, and J. L. Miranda-Sierra, "Propagation analysis and deployment of a wireless sensor network in a forest," Progress in Electromagnetics Research, vol. 106, pp. 121-145, 2010.

[8] J. Trubilowicz, K. Cai, and M. Weiler, "Viability of motes for hydrological measurement," Water Resources Research, vol. 46, no. 4, Article ID W00D22, 2010.

[9] Y. Kim, R. G. Evans, and W. M. Iversen, "Remote sensing and control of an irrigation system using a distributed wireless sensor network," IEEE Transactions on Instrumentation and Measurement, vol. 57, no. 7, pp. 1379-1387, 2008.

[10] B. D. Buckner, V. Markov, L. C. Lai, and J. C. Earthman,
"Laser-scanning structural health monitoring with wireless sensor motes," Optical Engineering, vol. 47, no. 5, Article ID 054402, 2008.

[11] V. Berisha, H. Kwon, and A. Spanias, "Real-time acoustic monitoring using wireless sensor motes," in Proceedings of IEEE International Symposium on Circuits and Systems (ISCAS '06), pp. 847-850, May 2006.

[12] C. U. Grosse, S. D. Glaser, and M. Krüger, "Initial development of wireless acoustic emission sensor Motes for civil infrastructure state monitoring," Smart Structures and Systems, vol. 6, no. 3, pp. 197-209, 2010.

[13] K. S. C. Kuang, S. T. Quek, and M. Maalej, "Remote flood monitoring system based on plastic optical fibres and wireless motes," Sensors and Actuators A, vol. 147, no. 2, pp. 449-455, 2008.

[14] Y. Z. Li, L. Wang, X. M. Wu, and Y. T. Zhang, "Experimental analysis on radio transmission and localization of a zigbeebased wireless healthcare monitoring platform," in Proceedings of the 5th International Conference on Information Technology and Applications in Biomedicine (ITAB '08), pp. 488-490, Nanjing, China, May 2008.

[15] S. Dagtas, G. Pekhteryev, Z. Sahinoglu, H. Cam, and N. Challa, "Real-time and secure wireless health monitoring," International Journal of Telemedicine and Applications, vol. 2008, Article ID 135808, 10 pages, 2008.

[16] Y. Rahal, H. Pigot, and P. Mabilleau, "Location estimation in a smart home: system implementation and evaluation using experimental data," International Journal of Telemedicine and Applications, vol. 2008, Article ID 142803, 9 pages, 2008.

[17] K. Främling, I. Oliver, J. Honkola, and J. Nyman, "Smart spaces for ubiquitously smart buildings," in Proceedings of the 3 rd International Conference on Mobile Ubiquitous Computing, Systems, Services, and Technologies (UBICOMM '09), pp. 295300, October 2009.

[18] K. McGlinn, R. Brennan, D. O'Sullivan, and D. Lewis, "The SimCon generator: an interactive context simulator for rapid evaluation of smart building applications using virtual reality," in Proceedings of the 8th IEEE International Workshop on Managing Ubiquitous Communications and Services, 2011.

[19] B. Bach, D. Wilhelmer, and P. Palensky, "Smart buildings, smart cities and governing innovation in the new millennium," in Proceedings of the 8th IEEE International Conference on Industrial Informatics (INDIN '10), pp. 8-14, July 2010.

[20] M. A. Zamora-Izquierdo, J. Santa, and A. F. Gomez-Skarmeta, "An integral and networked home automation solution for indoor ambient intelligence," IEEE Pervasive Computing, vol. 9, 2010.

[21] R. Yang and L. Wang, "Multi-objective optimization for decision-making of energy and comfort management in building automation and control," Sustainable Cities and Society, vol. 2, no. 1, pp. 1-7, 2012.

[22] M. Aiello and S. Dustdar, "Are our homes ready for services? A domotic infrastructure based on the Web service stack," Pervasive and Mobile Computing, vol. 4, no. 4, pp. 506-525, 2008.

[23] L. K. Haakenstad, "Open protocol standard for computerized building systems: BACnet," in Proceedings of the IEEE International Conference on Control Applications (CCA '99), pp. 15851590, August 1999.

[24] T. Teich, D. Szendrei, M. Schrader, F. Jahn, and S. Franke, 
"Feasibility of integrating heating valve drivers with KNXstandard for performing dynamic hydraulic balance in domestic buildings," Proceedings of World Academy of Science, Engineering and Technology, vol. 73, pp. 367-372, 2011.

[25] C. Bujdei and S. A. Moraru, "Ensuring comfort in office buildings: designing a KNX monitoring and control system," in Proceedings of the 7th International Conference on Intelligent Environments, 2011.

[26] T. J. Park and S. H. Hong, "Experimental case study of a BACnet-based lighting control system," IEEE Transactions on Automation Science and Engineering, vol. 6, no. 2, pp. 322-333, 2009.

[27] W. Guo, W. M. Healy, and M. Zhou, "ZigBee-Wireless Mesh Networks for building automation and control," in Proceedings of the International Conference on Networking, Sensing and Control (ICNSC '10), pp. 731-736, April 2010.

[28] X. Liu, T. Alpcan, and C. Bauckhage, "Adaptive wireless services for augmented environments," in Proceedings of the 6th Annual International Conference on Mobile and Ubiquitous Systems: Networking and Services (MobiQuitous '09), July 2009.

[29] Y. Sun, G. Zhao, and H. Luo, "Smart building control based on wireless sensor-actuator networks," Chinese Journal of Electronics, vol. 20, no. 3, pp. 437-442, 2011.

[30] C. H. Lu and L. C. Fu, "Robust location-aware activity recognition using wireless sensor network in an attentive home," IEEE Transactions on Automation Science and Engineering, vol. 6, no. 4, pp. 598-609, 2009.

[31] D. M. Han and J. H. Lim, "Smart home energy management system using IEEE 802.15.4 and ZigBee," IEEE Transactions on Consumer Electronics, vol. 56, no. 3, pp. 1403-1410, 2010.

[32] J. Byun and S. Park, "Development of a self-adapting intelligent system for building energy saving and context-aware smart services," IEEE Transactions on Consumer Electronics, vol. 57, no. 1, pp. 90-98, 2011.

[33] H. C. Tung, K. F. Tsang, L. L. Lai, K. L. Lam, and H. Y. Tung, "Hybrid energy management solution for smart building," in Proceedings of IEEE International Conference on Consumer Electronics (ICCE'11), pp. 509-510, January 2011.

[34] C. A. M. Bolzani, C. Montagnoli, and M. L. Netto, "Domotics over IEEE 802.15.4-a spread spectrum home automation application," in Proceedings of IEEE 9th International Symposium on Spread Spectrum Symposium on Spread Spectrum Techniques and Applications (ISSSTA '06), pp. 396-400, August 2006.

[35] V. Sulc, R. Kuchta, and R. Vrba, "IQRF smart house-a case study," in Proceedings of the 3rd International Conference on Advances in Mesh Networks (MESH '10), pp. 103-108, July 2010.

[36] J. P. Tae, J. C. You, K. P. Dong, and H. H. Seung, "BACnet over ZigBee, A new approach to wireless datalink channel for BACnet," in Proceedings of the 5th IEEE International Conference on Industrial Informatics (INDIN '07), pp. 33-38, June 2007.

[37] J. A. Nazabal, C. Fernandez-Valdivielso, F. Falcone, I. R. Matias, and S. C. Mukhopadhyay, "Integration of hybrid sensing networks in indoor intelligent homes," in Proceedings of the 5th International Conference on Sensing Technology (ICST '11), 2011.

[38] F. Österlind, E. Pramsten, D. Roberthson, J. Eriksson, N. Finne, and T. Voigt, "Integrating building automation systems and wireless sensor networks," in Proceedings of the 12th IEEE International Conference on Emerging Technologies and Factory
Automation (ETFA '07), pp. 1376-1379, September 2007.

[39] P. Yi, A. Iwayemi, and C. Zhou, "Building automation networks for smart grids," International Journal of Digital Multimedia Broadcasting, vol. 2011, Article ID 926363, 12 pages, 2011.

[40] S. Farahani, ZigBee Wireless Networks and Transceivers, Elsevier, 2008.

[41] D. Gislason, Zigbee Wireless Networking, Newnes, 2008.

[42] Y. Li, J. Maorong, G. Zhenru, Z. Weiping, and G. Tao, “Design of home automation system based on ZigBee wireless sensor network," in Proceedings of the 1st International Conference on Information Science and Engineering (ICISE '09), pp. 26102613, Nanjing, China, December 2009.

[43] M. Arai, H. Kawamura, and K. Suzuki, "Estimation of ZigBee's RSSI fluctuated by crowd behavior in indoor space," in Proceedings of SICE Annual Conference (SICE '10), pp. 696701, Taiwan, August 2010.

[44] R. M. Pellegrini, S. Persia, D. Volponi, and G. Marcone, "RF propagation analysis for ZigBee Sensor Network using RSSI measurements," in Proceedings of the 2nd International Conference on Wireless Communication, Vehicular Technology, Information Theory and Aerospace and Electronic Systems Technology, Wireless (VITAE'11), March 2011.

[45] L. Consolini, P. Medagliani, and G. Ferrari, "Adjacency matrixbased transmit power allocation strategies in wireless sensor networks," Sensors, vol. 9, no. 7, pp. 5390-5422, 2009.

[46] J. S. Lee, "Performance evaluation of IEEE 802.15.4 for lowrate wireless personal area networks," IEEE Transactions on Consumer Electronics, vol. 52, no. 3, pp. 742-749, 2006.

[47] H. Hashemi, "Indoor radio propagation channel," Proceedings of the IEEE, vol. 81, no. 7, pp. 943-968, 1993.

[48] J. Fink, N. Michael, A. Kushleyev, and V. Kumar, "Experimental characterization of radio signal propagation in indoor environments with application to estimation and control," in Proceedings of IEEE/RSJ International Conference on Intelligent Robots and Systems (IROS '09), pp. 2834-2839, St. Louis, Mo, USA, October 2009.

[49] J. M. Hernando, Transmisión por Radio, Universitaria Ramón Areces, Madrid, Spain, 5th edition, 2008.

[50] Y. Wang, W.-J. Lu, and H.-B. Zhu, "An empirical path-loss model for wireless channels in indoor short-range office environment," International Journal of Antennas and Propagation, vol. 2012, Article ID 636349, 7 pages, 2012.

[51] A. Gaugue, C. Lièbe, P. Combeau et al., "Ultra-wideband indoor channel modelling using ray-tracing software for through-the-wall imaging radar," International Journal of Antennas and Propagation, vol. 2010, Article ID 934602, 14 pages, 2010.

[52] A. E. Tümer and M. Gündüz, "Energy-efficient and fast data gathering protocols for indoor wireless sensor networks," Sensors, vol. 10, no. 9, pp. 8054-8069, 2010.

[53] M. F. Iskander and Z. Yun, "Propagation prediction models for wireless communication systems," IEEE Transactions on Microwave Theory and Techniques, vol. 50, no. 3, pp. 662-673, 2002.

[54] A. W. Reza, M. S. Sarker, and K. Dimyati, "A novel integrated mathematical approach of ray-tracing and genetic algorithm for optimizing indoor wireless coverage," Progress in Electromagnetics Research, vol. 110, pp. 147-162, 2010.

[55] H. D. Hristov, Fresnel Zones in Wireless Links, Zone Plate Lenses and Antennas, s.l, Artech House, 2000. 
[56] Recomendattion UIT-R P. 526-11, s.l., "Propagación por difracción. Serie P. Propagación de las ondas radioeléctricas, 10/2009".

[57] R. J. Luebbers, "Heuristic UTD slope diffraction coefficient for rough lossy wedges," IEEE Transactions on Antennas and Propagation, vol. 37, no. 2, pp. 206-211, 1989.

[58] R. J. Luebbers, "Comparison of lossy wedge diffraction coefficients with application to mixed path propagation loss prediction," IEEE Transactions on Antennas and Propagation, vol. 36, no. 7, pp. 1031-1034, 1988. 

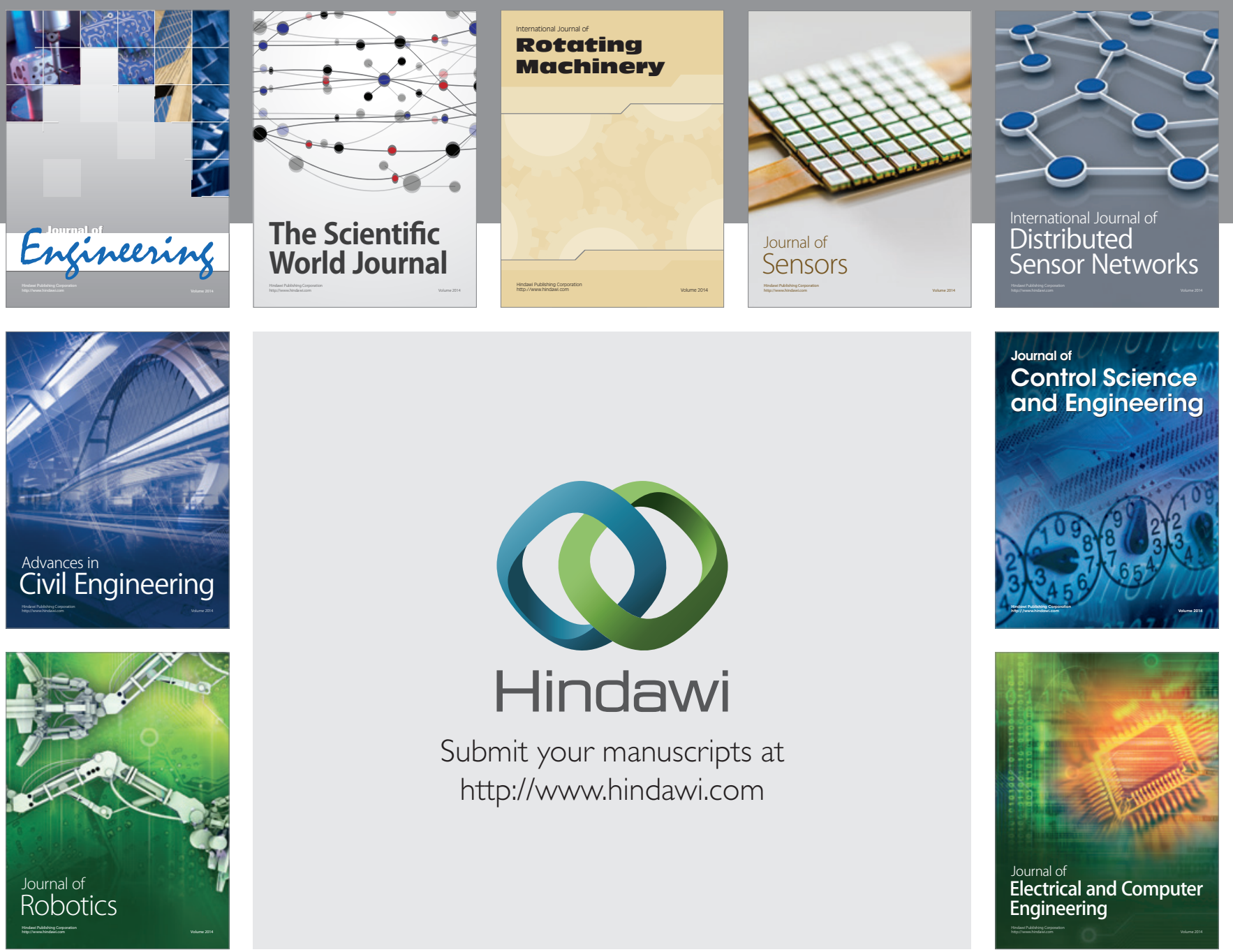

Submit your manuscripts at

http://www.hindawi.com
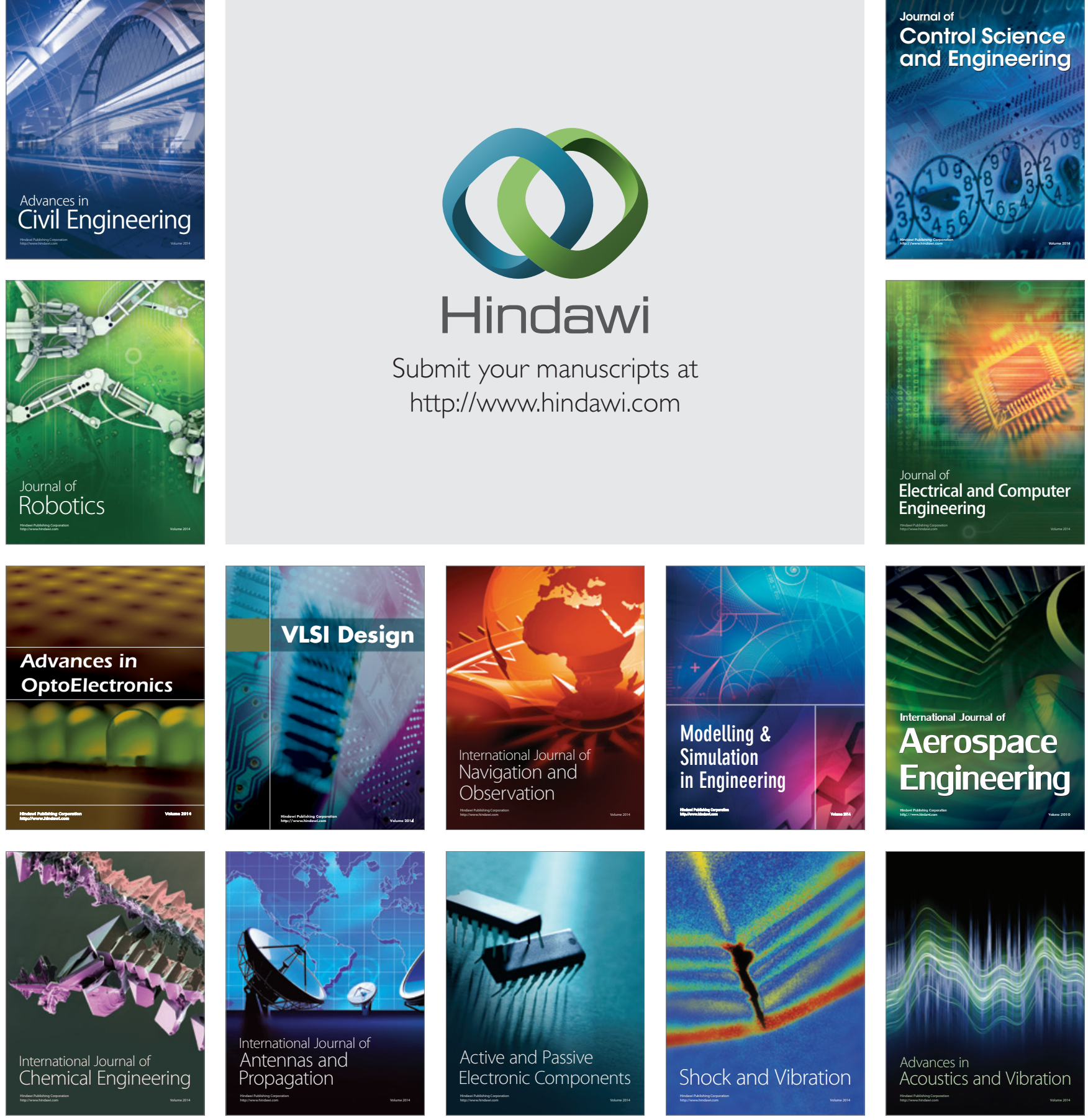